\title{
New Generation of Superconducting Nanowire Single-Photon Detectors
}

\author{
G.N. Goltsman a \\ Moscow State Pedagogical University, 1 Malaya Pirogovskaya, 119991, Moscow, Russia
}

\begin{abstract}
We present an overview of recent results for new generation of infrared and optical superconducting nanowire single-photon detectors (SNSPDs) that has already demonstrated a performance that makes them devices-of-choice for many applications. SNSPDs provide high efficiency for detecting individual photons while keeping dark counts and timing jitter minimal. Besides superior detection performance over a broad optical bandwidth, SNSPDs are also compatible with an integrated optical platform as a crucial requirement for applications in emerging quantum photonic technologies. By embedding SNSPDs in nanophotonic circuits we realize waveguide integrated single photon detectors which unite all desirable detector properties in a single device.
\end{abstract}

Keywords: superconducting single photon detectors, optical and IR wavelength radiation.

Routinely superconducting nanowire single-photon detector [1] is patterned from 4-nm-thick NbN film as 120 -nm-wide and meander-shaped strip that covers a square area of $10 \mathrm{x} 10 \mu \mathrm{m}^{2}$. At wavelength $\lambda \leq 1.5 \mu \mathrm{m}$ quantum efficiency (QE) of our devices approaches $30 \%$ at $2 \mathrm{~K}$ with $35 \mathrm{ps}$ timing jitter. The single-photon counting was observed at wavelength up to $5.6 \mu \mathrm{m}$ with QE of $\sim 1 \%$. Simultaneously, at $2 \mathrm{~K}$ the SNSPD has negligibly low dark counts of $2 \times 10^{-4} \mathrm{~s}^{-1}$ [2]. It provides NEP value of $10^{-20} \mathrm{~W} / \mathrm{Hz}^{1 / 2}$ at $\lambda \leq 1.3 \mu \mathrm{m}$ and $10^{-18} \mathrm{~W} / \mathrm{Hz}^{1 / 2}$ at $5 \mu \mathrm{m}$. Our investigations [3] allowed us to develop an SNSPD with photon-number resolving capability. Single-, two-, three- and four-photon absorptions were clearly observed. Finally, successful coupling of the SNSPD with a single mode optical fiber makes SNSPD a device of choice for wide range of applications including study of single-photon sources, telecom systems, quantum key distribution systems and quantum computing.

Besides application in classical optics it was realized that nanophotonic integrated circuits (NPICs) hold promise for more exotic use. Employing only waveguide based photonic devices the on-chip equivalents of free-space optical components can be implemented, offering a new route towards scalable linear optical quantum computing [4]. In this case NPICs are operated with single photons in contrast to the relatively high optical intensities used for telecommunication. However, for fully integrated quantum circuits not only passive devices but also active ingredients such as single photon sources and integrated single photon detectors are required [5]. A monolithic implementation of all building blocks of a quantum photonic circuit would then be able to overcome the stability and scalability limitations of bulk optic realizations.

\footnotetext{
${ }^{a}$ Corresponding author: goltsman@mspu-phys.ru
} 
Today, state-of-the-art SNSPDs are predominantly stand-alone units absorbing photons under normal incidence. Because the nanowire meanders are prepared from ultrathin films the optical absorption in a single pass is limited which in turn limits the overall detection efficiency. To increase photon absorption several approaches to optical cavity implementation have been realized recently. The most successful one allowed achievement of almost unity absorption in SNSPD meander [6]. Although cavity-integrated SNSPDs will surely evolve as a stand-alone detectors they are not very suitable for NPICs. Such difficulties can be overcome by realizing travelling wave superconducting nanowire detectors directly on top of nanophotonic waveguides [7-9]. In such a geometry photons propagate along the length of the superconducting nanowire. Therefore the interaction length can be increased to essentially achieve unity absorption efficiency. This in turn translates into high on-chip detection efficiency. Depending on the material system from which the waveguides are prepared different wavelength regimes can be targeted. In the case of silicon, a relatively small bandgap restricts the operation window to wavelengths above $1100 \mathrm{~nm}$. When broader wavelength access is required, silicon related materials such as silicon nitride (Si3N4) can be used to enhance the detection window for visible and near-infrared wavelengths [10]. In terms of superconducting materials suitable for SNSPD manufacture niobium nitride $(\mathrm{NbN})$ has been an attractive candidate [11].

Since the waveguide integrated detectors are fabricated with a small device footprint, the overall meander length is significantly shorter than in traditional fiber-coupled SNSPDs. This results in an equivalent reduction of the kinetic inductance of the detector and thus reduced reset time. For the silicon based detector devices we find a minimum relaxation time of $500 \mathrm{ps}$, which allows for single photon detection rates above $1 \mathrm{GHz}$. Furthermore, the devices provide very low timing jitter below $20 \mathrm{ps}$ which is attractive for on-chip time-resolved measurements [9].

Waveguide integrated single photon detectors are promising devices because they combine many desirable detector properties all in a single compact optical component. Particularly attractive is the high timing resolution, as well as the very low dark count rate and hence low noise-equivalent power. Because waveguide integrated single photon detectors can be fabricated with high yield and uniform performance across a large area, they are prime candidates for multi-detector architectures. With recent advances in on-chip single photon source manufacture all required elements for future quantum photonic networks can be monolithically prepared with prospect for emerging applications in optical quantum computing.

This work was supported by the Ministry of Education and Science of the Russian Federation (contract No. 14.586.21.0007; Project ID: RFMEFI58614X0007).

\section{References}

1. G. Goltsman, O. Okunev, G. Chulkova et. al., Appl. Phys. Lett. 79, No 6. P. 705-707 (2001)

2. A. Korneev, P. Kouminov, V. Matvienko, G. Chulkova, K. Smirnov, B. Voronov, G. N. Gol'tsman, Appl. Phys. Lett. 84, No 26. P. 5338-5340 (2004.)

3. A. Divochiy, F. Marsili, D. Bitauld, A. Gaggero, R. Leoni, F. Mattioli, A. Korneev, V. Seleznev, N. Kaurova, O. Minaeva, G. Gol'tsman, K. G. Lagoudakis, M. Benkhaoul, F. Levy, A. Fiore, Nat. Photon. 2. P. 302-306 (2008)

4. O'Brien J.L., Furusawa A., Vuckovic J., Nat. Photon. 3. P. 687-695 (2009)

5. R. H. Hadfield, Nat. Photon. 3. P. 696-705 (2009)

6. F. Marsili, V. B. Verma, J. A. Stern et. al., Nat. Photon. 7. P. 210-214 (2013)

7. J. P. Sprengers, et al., Appl. Phys. Lett. 99, P.181110-181112 (2011)

8. T. Gerrits et al., Phys. Rev. A. 84. P. 060301-060304 (2011)

9. W. H. P. Pernice, C. Schuck, O. Minaeva, M. Li, G.N. Goltsman, A.V. Sergienko, H.X. Tang, Nature communications. 3 (2012)

10. C. Schuck, W.H.P. Pernice, H.X. Tang, Scientific reports. 3 (2013)

11. A. Verevkin, J. Zhang et. al., Appl. Phys. Lett. 80, No 25. P. 4687-4689 (2002) 